\title{
AKT/mTOR substrate P70S6K is frequently phosphorylated in gallbladder cancer tissue and cell lines
}

\author{
This article was published in the following Dove Press journal: \\ OncoTargets and Therapy \\ 2 October 2013 \\ Number of times this article has been viewed
}

\author{
Pamela Leal',* \\ Patricia Garcia ${ }^{2, *}$ \\ Alejandra Sandoval' \\ Kurt Buchegger' \\ Helga Weber' \\ Oscar Tapia \\ Juan C Roa ${ }^{1,2}$ \\ 'Department of Pathology, \\ Universidad de La Frontera, Center \\ of Genetical and Immunological \\ Studies-Scientific and Technological \\ Bioresource Nucleus, Temuco, \\ ${ }^{2}$ Department of Pathology, Pontificia \\ Universidad Catolica de Chile, \\ Santiago, Chile \\ *These authors contributed equally \\ to this paper
}

Background: Gallbladder carcinoma is a highly malignant tumor and a public health problem in some parts of the world. It is characterized by a poor prognosis and its resistance to radio and chemotherapy. There is an urgent need to develop novel therapeutic alternatives for the treatment of gallbladder carcinoma. The mammalian target of the rapamycin (mTOR) signaling pathway is activated in about $50 \%$ of human malignancies, and its role in gallbladder carcinoma has previously been suggested. In the present study, we investigated the phosphorylation status of the mTOR substrate p70S6K in preneoplastic and neoplastic gallbladder tissues and evaluated the effect of three mTOR inhibitors on cell growth and migration in gallbladder carcinoma cell lines.

Methods: Immunohistochemical staining of phospho-p70S6K was analyzed in 181 gallbladder carcinoma cases, classified according to lesion type as dysplasia, early carcinoma, or advanced carcinoma. Protein expression of AKT/mTOR members was also evaluated in eight gallbladder carcinoma cell lines by Western blot analysis. We selected two gallbladder carcinoma cell lines (G415 and TGBC-2TKB) to evaluate the effect of rapamycin, RAD001, and AZD8055 on cell viability, cell migration, and protein expression.

Results: Our results showed that phospho-p70S6K is highly expressed in dysplasia $(66.7 \%$, $12 / 18)$, early cancer $(84.6 \%, 22 / 26)$, and advanced cancer $(88.3 \%, 121 / 137)$. No statistical correlation was observed between phospho-p70S6K status and any clinical or pathological features, including age, gender, ethnicity, wall infiltration level, or histological differentiation $(P<0.05)$. In vitro treatment with rapamycin, RAD001, and AZD8055 reduced cell growth, cell migration, and phospho-p70S6K expression significantly in G-415 and TGBC-2TKB cancer cells $(P<0.001)$.

Conclusion: Our findings confirm the upregulation of this signaling pathway in gallbladder carcinoma and provide a rationale for the potential use of mTOR inhibitors as a therapeutic strategy for human gallbladder carcinoma.

Keywords: gallbladder cancer, p70S6K, mTOR inhibitor, AKT, migration, cell line

\section{Introduction}

Gallbladder cancer (GBC) is the most common neoplasia of the biliary tract and it is characterized by high mortality and a poor prognosis. ${ }^{1}$ According to the Chilean Department of Health Statistics (http://www.deis.cl), GBC is the second leading cause of cancer death among women, with a mortality rate only slightly lower than that of breast cancer. Most GBCs are diagnosed incidentally after a cholecystectomy, and unfortunately it is usually at an advanced stage. ${ }^{2}$ Strategies to improve overall survival of patients with advanced GBC include the use of adjuvant radiotherapy and chemotherapy with 5-fluorouracil, gemcitabine, and platinum analogs, which have been
Correspondence: Juan Carlos Roa Department of Pathology, School of Medicine, Universidad de La Frontera, Manuel Montt I 2, PO Box 54-D,

Temuco, Chile

Tel +5645325792

Fax +5645325730

Email jcroa@ufro.cl 
studied as single agents or in combination. ${ }^{3-5}$ On the basis of a Phase III clinical trial, ${ }^{6}$ the combination of gemcitabine and cisplatin is considered the standard for treating patients with unresectable, metastatic biliary tract cancers. ${ }^{7}$ However, despite the statistically significant higher response rate and improved overall survival (compared with a gemcitabineonly regimen), there are frequent adverse events arising from these drugs.

In the last few years, molecular targeted therapy has emerged as a promising strategy for the treatment of advanced GBC and other biliary tract carcinomas. Different molecularly targeted cancer drugs have been evaluated in patients with advanced biliary tract carcinomas in Phase I/II studies, either in monotherapy or in combination with other chemotherapeutic or targeted agents. ${ }^{8,9}$ Among these are bevacizumab, the humanized monoclonal antibody against VEGF (vascular endothelial growth factor), ${ }^{10,11}$ sorafenib, a multikinase inhibitor that targets BRAF (a serine/threonine-specific protein kinase) and VEGFR (VEGF receptor 1, 2 and 3), ${ }^{12}$ and erlotinib ${ }^{10,13,14}$ and cetuximab, ${ }^{15}$ which are small-molecule inhibitors against the epithelial growth factor receptor. The results of these trials highlight the clinical relevance of targeted therapy in the management of biliary tract carcinomas, but there is no conclusive evidence to determine its efficacy in GBC because studies frequently group together different biliary tract tumors which should be considered separately due to their genetic heterogeneity.

In addition to VEGF and EGFR (epithelial growth factor receptor), other molecules could also be interesting "druggable" targets for GBC. Use of inhibitors against the mTOR signaling pathway have been proposed as an attractive therapeutic strategy based on the fact that this pathway seems to be activated in $\mathrm{GBC},{ }^{16}$ and on preclinical data showing that the mTOR inhibitor, rapamycin, can reduce the incidence of gallbladder carcinoma in a transgenic mouse model. ${ }^{17}$ However, to our knowledge and according to ClinicalTrials. gov, ${ }^{18}$ there is currently only one clinical study evaluating the effectiveness and safety of mTOR pathway inhibitors in biliary tract carcinomas, including some GBC cases in the same category. This is a Phase II clinical trial conducted to evaluate the use of an AKT inhibitor (MK2206) in patients with advanced refractory biliary cancer (ClinicalTrials.gov ID NCT01425879). ${ }^{19}$

mTOR is a serine/threonine kinase, the catalytic subunit of two signaling complexes called mTORC1 and mTORC2. Both contain common and specific partner proteins: mTORC1 contains RAPTOR, mLST8, and PRAS40; mTORC2 also contains mTOR and mLST8 but is defined by the unique regulatory proteins RICTOR, mSIN1, and PROTOR. Both mTORC1 and mTORC2 additionally interact with DEPTOR, which inhibits their activities. ${ }^{20}$ Growth factors and nutrients, such as vitamins and amino acids, promote mTORC1 signaling through phosphorylation of eukaryotic initiation factor 4E-binding protein 1 (4E-BP1) and ribosomal S6 kinase 1 (S6K1), which are the best known downstream effectors of mTOR. The regulation and function of mTORC2 are less understood, but its role is recognized as a mediator of actin cytoskeletal organization and cell polarization, and in the phosphorylation of AGC kinase family members, which regulate cell survival, cell cycle progression, and anabolism. Further, mTORC2 activity has been linked to mTORC1 via direct phosphorylation of RICTOR by S6K1. ${ }^{21}$

A main pathway that signals through mTORC1 is the phosphoinositide 3 kinase (PI3K)/AKT (v-Akt murine thymoma viral oncogene homolog-1) signal transduction pathway, which is critically involved in mediation of cell survival and proliferation. ${ }^{22}$ AKT phosphorylates mTOR directly, and activation of $\mathrm{mTORC} 1$ results in phosphorylation of several downstream targets. The best characterized downstream substrates of mTORC1 are 4E-BP1 and the ribosomal protein p70S6K or S6K1. Phosphorylation of 4E-BP1 releases eIF4E, allowing the initiation of translation. By acting on S6K, mTOR facilitates ribosome biogenesis and translation elongation. ${ }^{23}$ The phosphorylation status of p70S6K is commonly used as a marker of mTOR activity and for pharmacodynamic monitoring of mTOR inhibition. ${ }^{24}$

Since mTOR plays a central role in cell growth, proliferation, and survival, and since it is frequently upregulated in tumor cells, thus contributing to tumor growth, angiogenesis, and metastasis, ${ }^{25-29} \mathrm{mTOR}$ represents an ideal target in cancer therapy. Indeed, the efficacy and safety of different mTOR inhibitors have been evaluated in preclinical and clinical trials across a wide range of cancers. ${ }^{21}$ The anticancer efficacy of rapalogs (rapamycin analogs) and small inhibitors (adenosine triphosphate [ATP]-competitive inhibitors) has been evaluated in monotherapy and combination therapy. Among them, everolimus (RAD001), an orally administered rapamycin analog, has shown promising results in experimental studies, inhibiting tumor growth and having antiangiogenic effects, ${ }^{30-32}$ and has been approved by the US Food and Drug Administration for the treatment of renal cell carcinoma refractory to inhibitors of VEGF receptor signaling. ${ }^{33}$ Although rapalogs have more favorable pharmacokinetic and solubility properties than rapamycin, they are only able to inhibit mTORC1 signaling. By contrast, 
the new generations of mTOR inhibitors, which bind to the ATP binding site of mTOR, inhibit the catalytic activity of mTORC1 and mTORC2. ${ }^{34}$ The anticancer efficacy of these inhibitors reported in preclinical evaluation has been superior to that of rapamycin analogs. ${ }^{35-38}$

As mentioned previously, the mTOR signaling pathway seems to be activated in human GBC. We have previously shown that phosphorylation of mTOR is frequent in gallbladder tumors and it is associated with a poorer prognosis in patients with advanced GBC. ${ }^{16}$ In order to validate mTOR pathway activation in $\mathrm{GBC}$, here we first investigated the phosphorylation status of the mTOR substrate, p70S6K, in preneoplastic and neoplastic gallbladder tissues by immunohistochemistry. We then evaluated the activation of the AKT/mTOR pathway and its major downstream components in GBC cell lines, as well as the effect of three mTOR inhibitors (rapamycin, RAD001, and AZD8055) on both cell growth and migration. Our findings confirm mTOR pathway activation in GBC tumors according to immunohistochemical analysis of phosphorylated p70S6K and in vitro assays in GBC cell lines.

\section{Materials and methods Immunohistochemical expression of phospho-p70S6K}

A total of 181 gallbladder tissues from patients who underwent curative surgical resection at Hernan Henriquez Aravena Hospital (Temuco, Chile) between 1987 and 2010 were included in this study. Tissue microarrays were built and samples were classified according to lesion type as dysplasia $(n=18)$, early carcinoma $(n=26)$, or advanced carcinoma $(n=137)$. Three different representative areas per case were sampled. None of these patients had received preoperative chemotherapy. Our study was approved by the ethics committee of the School of Medicine, Universidad de La Frontera, and all patients provided written informed consent for use of their tumor tissue in clinical research. Phospho-p70S6K expression was evaluated using a previously described immunohistochemistry protocol. ${ }^{39}$ Briefly, sections $4 \mu \mathrm{m}$ thick were deparaffinized with xylene and rehydrated through an alcohol gradient. For epitope retrieval, specimens were heated $\left(95^{\circ} \mathrm{C}\right)$ for 30 minutes in citrate buffer $0.1 \mathrm{M}(\mathrm{pH}$ 6.0) in a pressure cooker. To reduce nonspecific background staining, the slides were incubated in 3\% hydrogen peroxide $\left(\mathrm{H}_{2} \mathrm{O}_{2}\right)$ for 10 minutes. After incubation with Ultra V Block (UltraVision LP detection system, Lab Vision Corporation, Fremont, CA, USA) for 10 minutes at room temperature, the tissues were incubated overnight at $4^{\circ} \mathrm{C}$ with a mouse monoclonal anti-phospho-p70S6K $\alpha$ (A-6, Santa Cruz Biotechnology Inc, Santa Cruz, CA, USA) in a dilution of 1:50. Labeling was detected with the DAB-Chromogen system (Dako North America Inc, Carpinteria, CA, USA) according to the manufacturer's protocol. After counterstaining with Harris' hematoxylin, the section was dehydrated, cleared, and mounted. We used gastric cancer tissue as the positive control and omission of the primary antibody was used as a negative control. The immunohistochemical staining of phosphop70S6K was examined by two independent and specialized pathologists (JCR, OT) without any information about clinicopathological features or prognosis. Immunohistochemical staining was evaluated based on staining intensity and positive cells within the whole tissue section using a previously described scoring system. ${ }^{40}$ The phospho-p70S6K expression level was evaluated by an $\mathrm{H}$ score, ${ }^{41}$ which was calculated by multiplying the percentage of positive cells by the corresponding staining intensity (1, weak; 2, moderate; and 3, strong), giving a maximum score of $300(100 \% \times 3)$. H scores $>50$ were considered positive.

\section{Cell lines}

$\mathrm{AKT} / \mathrm{mTOR} / \mathrm{p} 70 \mathrm{~S} 6 \mathrm{~K}$ pathway expression was evaluated in eight human GBC cell lines, ie, GB-d1, G-415, SNU-308, OCUG-1, NOZ, TGBC-1TKB, TGBC-2TKB, and TGBC24TKB. GB-d1 and SNU-308 were provided by Anirban Maitra (Department of Pathology, Johns Hopkins University School of Medicine, Baltimore, MD, USA); OCUG-1 and NOZ were obtained from the Health Science Research Resources Bank (Osaka, Japan); and G-415, TGBC-1TKB, TGBC-2TKB, and TGBC-24TKB were purchased from RIKEN BioResource Center (Ibaraki, Japan).

\section{Cell culture growth conditions}

G-415, GB-d1, and SNU-308 were grown in Roswell Park Memorial Institute (RPMI) 1640 medium (Thermo Scientific HyClone, Logan, UT, USA) supplemented with $10 \%$ fetal bovine serum, 10 units/mL penicillin and $10 \mathrm{mg} /$ $\mathrm{mL}$ streptomycin (1\% penicillin/streptomycin, Thermo Scientific HyClone). OCUG-1 was cultured in Dulbecco's Modified Eagle's Medium (high glucose) and NOZ in William's E medium (Invitrogen, Life Technologies Corporation, Grand Island, NY, USA) with $10 \%$ fetal bovine serum and $1 \%$ penicillin/streptomycin. TGBC-1TKB and TGBC-2TKB were grown in Dulbecco's Modified Eagle's Medium (high glucose) supplemented with 5\% fetal bovine serum and $1 \%$ penicillin/streptomycin. All eight cell lines were incubated at $37^{\circ} \mathrm{C}$ in a humidified 
atmosphere containing $5 \% \mathrm{CO}_{2}$ and subcultured during the logarithmic phase.

\section{mTOR inhibitors}

LY294002 inhibitor (\#9901) was purchased from Cell Signaling Technology (Danvers, MA, USA). Rapamycin (sirolimus, \#S1039), everolimus (RAD001, \#S1120), and AZD8055 (\#S1555) were purchased from Selleck Chemicals (Houston, TX, USA). All inhibitors were dissolved at $10 \mathrm{mM}$ in dimethylsulfoxide as stock solutions and stored at $-20^{\circ} \mathrm{C}$. All inhibitors were diluted in culture medium before each experiment and $0.1 \%$ dimethylsulfoxide in culture medium was used as a vehicle control.

\section{Antibodies}

Rabbit polyclonal antibodies purchased from Cell Signaling Technology were used for Western blot analysis as follows: anti-AKT (\#4685), anti-phospho-AKT(Ser 473, \#4060), antieIF4E (\#2067), anti-phospho-eIF4E (Ser209, \#9741), anti4E-BP1 (\#9644), anti-phospho-4E-BP1 (Thr37/46, \#2855), anti-p70S6K (\#2708), anti-phospho-p70S6K (Thr389, $\# 9234)$ and anti- $\beta$-actin (\#4970). The secondary antibody was goat anti-rabbit IgG horseradish peroxidase (Santa Cruz Biotechnology Inc). Mouse monoclonal anti-phospho-p70S6K antibody (\#sc8416) used for immunohistochemical analysis was purchased from Santa Cruz Biotechnology Inc.

\section{Western blot analysis}

For inhibition assay, cells were seeded on $25 \mathrm{~cm}^{2}$ culture plates in triplicate at a density of $3 \times 10^{5}$ cells. After a 4-hour attachment period, the cells were treated with LY294002 $(10 \mu \mathrm{M})$, rapamycin (50 nM), RAD001 (1 nM), AZD8055 (25 nM) and $0.1 \%$ dimethylsulfoxide (as control) for 18 hours. Cell lysate from gallbladder cells was prepared using RIPA buffer (SigmaAldrich Co, St Louis, MO, USA). Briefly, cells at 70\%-80\% confluency were washed three times with cold phosphate-buffered saline (PBS) and lysed on ice with RIPA buffer containing phenylmethylsulfonyl fluoride, protease and phosphatase inhibitor cocktail (Sigma-Aldrich). Whole cell lysate was collected after centrifugation at $8,000 \mathrm{~g}$ for 10 minutes at $4^{\circ} \mathrm{C}$. Protein concentrations were determined using a BCA Protein Assay Kit (Pierce, Thermo Fisher Scientific Inc, Rockford, IL, USA) according to the manufacturer's instructions. Equal amounts of total cellular protein $(30 \mu \mathrm{g})$ were separated by sodium dodecyl sulfate-polyacrylamide gel electrophoresis in $4 \%-12 \%$ NuPAGE $^{\circledR}$ Bis-Tris Precast Gels (Novex, Life Technologies Corporation) and electrotransferred to polyvinylidene difluoride membranes (Immobilon ${ }^{\circledR}-\mathrm{P}$ membrane,
Millipore, Bedford, MA, USA). The membranes were blocked with $1 \times$ Tris-buffered saline containing $0.05 \%$ Tween (TBST) and $5 \%$ fat-free milk for 1 hour at room temperature and incubated overnight at $4{ }^{\circ} \mathrm{C}$ with primary antibodies. After washing with TBST, the membranes were further incubated with the corresponding horseradish peroxidase-conjugated secondary antibodies for 1 hour at room temperature. Antibody-bound protein bands were detected with enhanced chemiluminescence reagent SuperSignal West Pico Substrate (Pierce) and photographed with Amersham Hyperfilm ECL autoradiography film (GE Healthcare Biosciences, Pittsburgh, PA, USA). $\beta$-actin expression was used as a loading control.

\section{Cell viability assay}

Cell viability was analyzed by MTS assay using CellTiter $96^{\circledR}$ AQueousOne Solution Reagent assay according to the manufacturer's instructions (Promega Corporation, Madison, WI, USA). G-415 and TGBC-2TKB cell lines were seeded on 96-well culture plates in triplicate at a density of $3.5 \times 10^{3}$ cells per well $(100 \mu \mathrm{L}$ medium/well). After an overnight attachment period, cells were treated with LY294002 $(10 \mu \mathrm{M})$, rapamycin (50 nM), RAD001 (1 nM), AZD8055 (25 nM), and 0.1\% dimethylsulfoxide (as control). After 24, 48, and 72 hours, $20 \mu \mathrm{L}$ of MTS solution were added to each well and the cells were incubated for an additional 1 hour at $37^{\circ} \mathrm{C}$. Cell viability was determined by measuring absorbance at $490 \mathrm{~nm}$ using a multiwell plate reader (Autobio Labtec Instruments Co, Ltd, Zhengzhou City, People's Republic of China). Viability percent was calculated using the following formula:

$$
\text { Viability }(\%)=(\mathrm{X} * 100 \%) / \mathrm{Y}
$$

where $\mathrm{X}$ is the absorbance of treated cells and $\mathrm{Y}$ is the absorbance of untreated cells.

\section{Cell migration assay}

Assays were performed using 24-well Transwell ${ }^{\mathrm{TM}}$ plates containing polycarbonate filters with an $8 \mu \mathrm{m}$ pore size (BD Biosciences, Bedford, MA, USA). Before seeding, cells were exposed to LY294002 $(10 \mu \mathrm{M})$, rapamycin (50 nM), RAD001 (1 nM), AZD8055 (25 nM), and 0.1\% dimethylsulfoxide (as control) for 1 hour at $37^{\circ} \mathrm{C}$. Complete RPMI 1640 medium was then placed in the lower chamber and $2.5 \times 10^{4}$ cells suspended in RMPI 1640 without fetal bovine serum were seeded into the upper chamber. After 24 hours, the cells were fixed in methanol for 15 minutes and then stained with $0.05 \%$ crystal violet in $25 \%$ methanol/PBS for 15 minutes. Cells on top of the membrane were removed using a cotton swab, and the 
filters were washed with PBS. Cells on the underside of the filters were viewed and counted under a microscope in 16 randomly selected fields.

\section{Statistical analysis}

All statistical analyses were performed using Statistical Package for the Social Sciences version 17 software (SPSS Inc., Chicago, IL, USA). The association between phosphop70S6K expression level and clinicopathological variables was examined using a Pearson's chi-square test or a Fisher's exact probability test, with $P<0.05$ being considered statistically significant. For cell viability and migration assays, differences between groups (treated and control) were examined using a two-way analysis of variance and Bonferroni post hoc test, with $P<0.001$ being considered statistically significant. Data were presented as the mean \pm standard error of the mean from at least three independent experiments.

\section{Results}

\section{Phospho-p70S6K is highly expressed in patients with advanced GBC}

In order to establish whether AKT/mTOR downstream serine/ threonine kinase p70S6K is frequently activated in GBC, we examined the expression of phospho-p70S6K (Thr389) by immunohistochemistry on tissue microarrays containing dysplasia $(n=18)$, early carcinoma $(n=26)$, or advanced carcinoma $(\mathrm{n}=137)$. Phospho-p70S6K immunohistochemistry expression was detectable with a uniform pattern in the nucleus of epithelial cells throughout all different tissue microarray cores. As a reference control, phospho-p70S6K expression was also evaluated in a few cases of chronic cholecystitis, which were mostly negative (Supplementary Figure S1). All clinicopathological features were obtained from medical records and are summarized in Supplementary Table S1. Briefly, 26 cases were classified as pT1a-pT1b (mucosal or muscular layer infiltration) and 137 as pT2-pT4 (subserosal or serosal infiltration). According to histological differentiation, 23 cases were classified as well differentiated, 79 as moderately differentiated, and 50 as poorly differentiated. As shown in Figure 1A-D, phospho-p70S6K was positively immunostained in the cytoplasm of gallbladder epithelial cells. As shown in Figure 1E, phospho-p70S6K was highly expressed in dysplasia $(66.7 \%, 12 / 18)$, early cancer (84.6\%, 22/26), and advanced cancer $(88.3 \%, 121 / 137)$.

Statistical significance was found between dysplasia and advanced cancer $(P<0.05)$. No statistically significant correlation was observed between phospho-p70S6K expression and any clinical or pathological variable, including
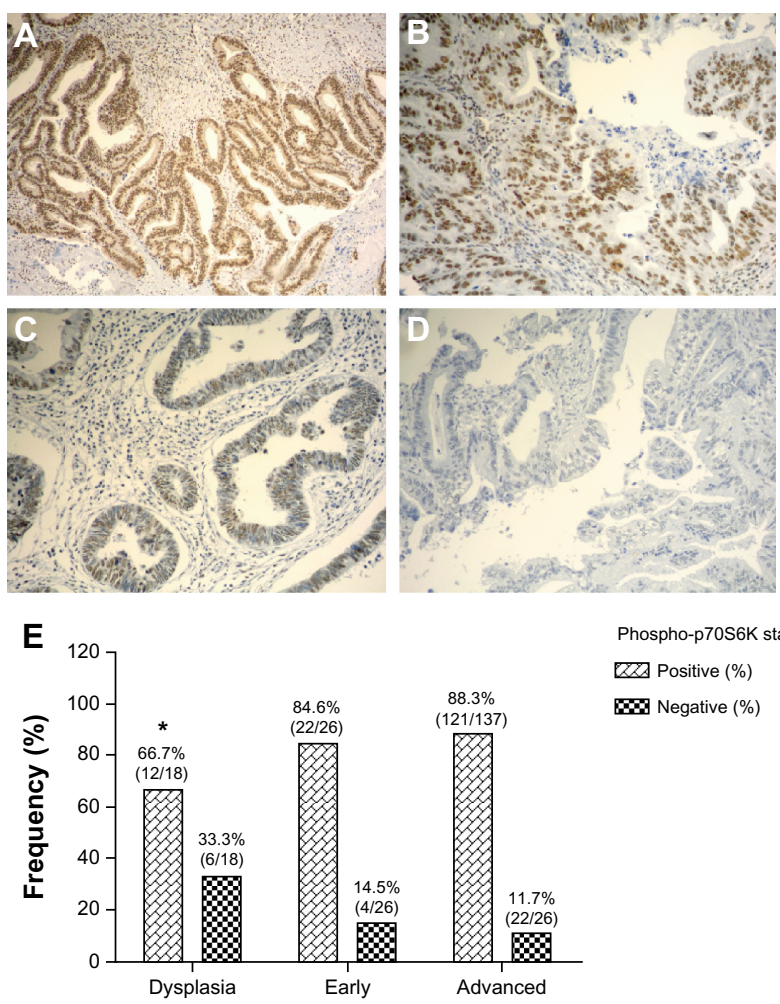

Phospho-p70S6K status QZ7 Positive (\%) W Negative (\%)

Figure I Immunohistochemical staining of phospho-p70S6K in gallbladder tissue. Representative images from gallbladder lesions showing intense (A), moderate (B), weak (C), and negative intensity (D) staining of phospho-p7056K. Images represent original magnification $100 \times$. (E) Frequency distribution for positive staining of phospho-p70S56K in sequential gallbladder lesions.

Note: ${ }^{*}$ Compared with advanced gallbladder cancer $(P<0.05)$.

age, gender, ethnicity, wall infiltration level, or histological differentiation (Supplementary Table S1).

\section{AKT/mTOR pathway is activated in human GBC cell lines}

In order to validate the activation of mTOR pathway observed in GBC tissues by immunohistochemistry, we examined the AKT/mTOR pathway and its major downstream components in eight GBC cell lines (GB-d1, G-415, SNU-308, OCUG-1, NOZ, TGBC-1TKB, TGBC-2TKB, and TGBC-24TKB) by Western blot. As shown in Figure 2, AKT/mTOR pathway members are expressed differentially in human GBC cell lines. Total AKT protein was detected strongly in five cell lines, while phospho-AKT (Ser473) was only detected in G-415, TGBC-1TKB, and TGBC-2TKB. All cell lines expressed similar amounts of total p70S6K and eIF4E proteins. In contrast, phospho-p70S6K (Thr389) expression was highly upregulated in TGBC-2TKB compared with the other cell lines. Phospho-eIF4E was undetectable in this cell panel. According to the constitutive activation status of AKT/mTOR pathways detected by Western blot, only 


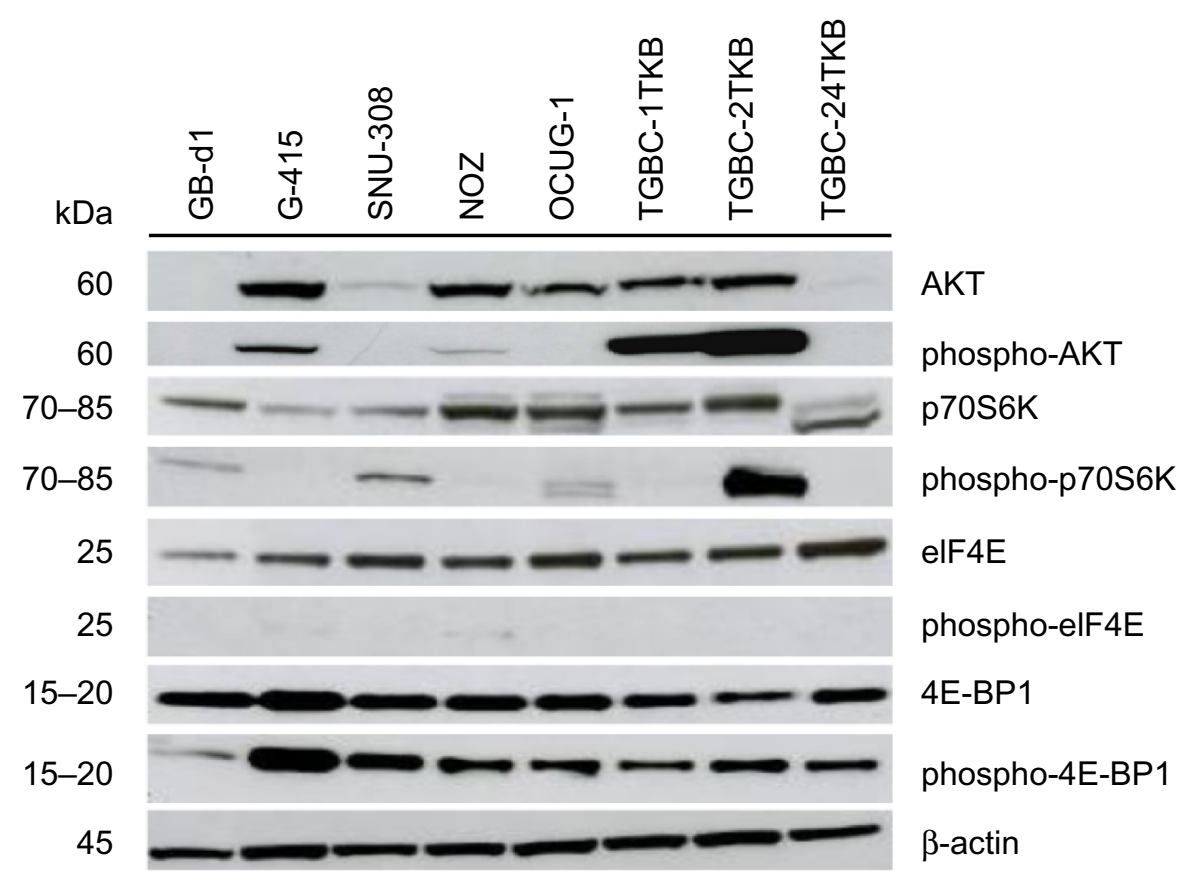

Figure 2 Activation of AKT/mTOR pathway in human gallbladder cancer cell lines. Total protein (35 $\mu \mathrm{g})$ from eight gallbladder cancer cell lines were subjected to immunoblotting using antibodies directed against AKT, phospho-AKT, p70S6K, phospho-p70S6K, elF4E, phospho-elF4E, 4E-BPI, and phospho-4E-BPI. Protein loading was normalized using an antibody recognizing $\beta$-actin.

Abbreviations: kDa, kiloDalton; $\beta$-actin, beta actin; AKT, protein kinase B; mTOR, mammalian target of rapamycin; p70S6K, p70S6 kinase; elF4E, eukaryotic translation initiation factor 4E; 4E-BPI, eukaryotic translation initiation factor 4E-binding protein I.

G-415 and TGBC-2TKB were selected for the following inhibition assays.

\section{Exposure to $\mathrm{mTOR}$ inhibitors decreases phosphorylation of AKT/mTOR downstream proteins}

To examine the inhibitory effect of mTOR inhibitors on G-415 and TGBC-2TKB protein expression, cells were exposed to LY294002 $(10 \mu \mathrm{M})$, rapamycin $(50 \mathrm{nM})$, RAD001 (1 nM), AZD8055 (25 nM), and 0.1\% dimethylsulfoxide (as control) for 18 hours. Cells were lysed and analyzed by Western blot using commercial antibodies. As shown in Figure 3, treatment with either mTOR inhibitor decreased the phosphorylated status of p70S6K in both cell lines markedly, although a slight activation of total $\mathrm{p} 70 \mathrm{~S} 6 \mathrm{~K}$ was observed in TGBC-2TKB. No significant changes were observed in total AKT and 4E-BP1 protein expression under the treatment conditions assayed. Similar results were also observed in the G-415 cell line.

\section{Effects of ATP-competitive inhibitors of $m T O R$ on cell viability and cell migration}

The inhibitory effect of various mTOR inhibitors, including LY294002 (an PI3K inhibitor) as a positive control, were tested on G-415 and TGBC-2TKB cells. Cell viability was analyzed by MTS assay according to the manufacturer's protocol. Cells were treated with increasing concentrations of LY294002, rapamycin, RAD001, and AZD8055 for 24, 48, and 72 hours. As shown in Figure 4, cell growth was significantly decreased by each inhibitor, concentration, or cell line used $(P<0.001)$. However, after 72 hours of drug treatment, even at the lowest concentration used, rapamycin and AZD8055 inhibitors showed the highest inhibition rate compared with the control ( $0.1 \%$ dimethylsulfoxide). The respective inhibition rates were as follows: G-415 (44.2\% and $43.4 \%)$ and TGBC-2TKB (48.1\% and $46.1 \%)$.

In order to establish the inhibitory effect of each inhibitor on cell migration, G-415 and TGBC-2TKB were exposed to $0.1 \%$ dimethylsulfoxide (as control), LY294002 (10 $\mu \mathrm{M})$, rapamycin (50 nM), RAD001 (1 nM), and AZD8055 (25 nM) for 1 hour. All concentrations evaluated corresponded to the minimum used for each inhibitor in cell viability assays, where a significant difference was observed compared with the untreated control. After 24 hours, the migration rate was significantly lower in treated cells than in untreated cells $(P<0.001)$. In the same way, suppression of cell migration was significantly greater in rapamycin and AZD8055 compared with LY294002 or RAD001 (Figure 5 and Supplementary Figure S2). Relative migration rates observed in 


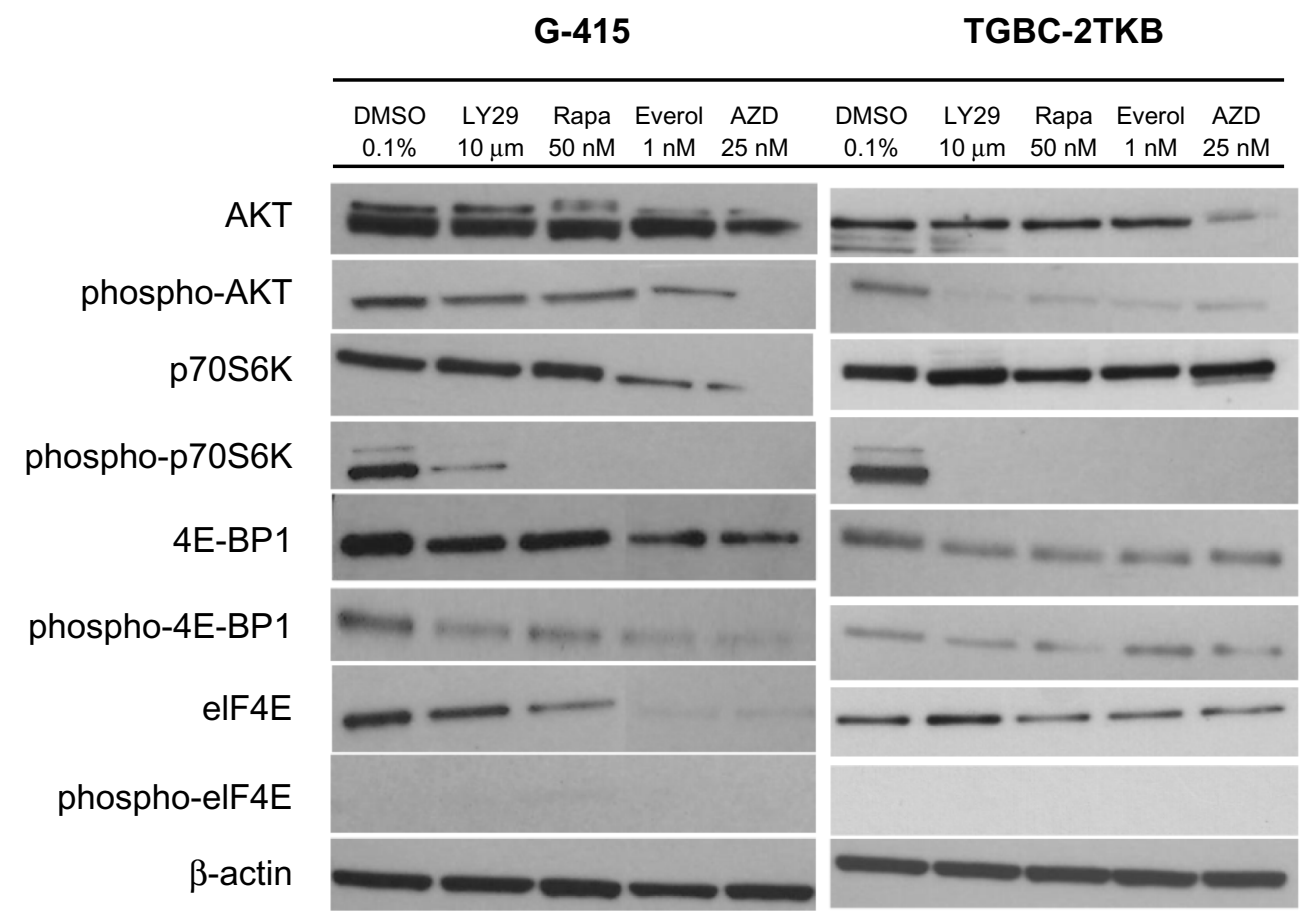

Figure 3 Effect of $m$ TOR inhibitors of $m$ TOR on AKT/mTOR signaling pathway in two gallbladder cancer cell lines. G-4I5 and TGBC-2TKB cells were grown in Roswell Park Memorial Institute I640 medium supplemented with I0\% fetal bovine serum and treated with LY294002 (I0 $\mu$ M), rapamycin (50 nM), RAD00 I (I nM), and AZD5085 $(25 \mathrm{nM})$ for 18 hours. Control cells received an equivalent amount of solvent ( $0.1 \%$ dimethylsulfoxide). Western blot analysis was carried out using antibodies against the total and phosphorylated portion of AKT, p70S6K, 4E-BPI, and elF4E proteins.

Abbreviations: DMSO, dimethyl sulfoxide; LY29, LY294002 PI3 kinase inhibitor; Rapa, rapamycin; Everol, everolimus; AZD, AZD-8055.

G-415 were $40.6 \%$ (rapamycin) and 36.9\% (AZD8055), while TGBC-2TKB showed a relative migration rate lower than G-415, with 28\% (rapamycin) and 9.6\% (AZD8055), respectively.

\section{Discussion}

mTOR is a highly conserved serine/threonine protein kinase that integrates both intracellular and extracellular signals and serves as a central regulator of cell metabolism, growth, proliferation, and survival. ${ }^{42}$ mTOR exerts its different biological functions primarily through forming two complexes, mTORC1 and mTORC2, both characterized by their association with essential partner proteins, which regulate kinase specificity towards distinct downstream targets..$^{20}$ mTORC 1 is best characterized by its ability to regulate cell growth and protein translation via phosphorylation of p70S6K1 and 4E-BPs. Meanwhile, mTORC2 is a mediator of actin cytoskeletal organization and cell polarization, and is also the critical kinase for AKT Ser473 phosphorylation. ${ }^{21}$

Deregulation of the mTOR signaling pathway has been associated with the pathogenesis of various human cancers, and several studies have shown its role in angiogenesis, tumor growth, and metastasis. ${ }^{25-29} \mathrm{Wu}$ et al were the first to suggest that the AKT/mTOR pathway is activated in human GBC. They reported that both phospho-AKT and phospho-mTOR levels are significantly increased in human adenocarcinoma (74.1\% and $92.6 \%$, respectively) compared with normal human gallbladder specimens (28.6\%). The authors also demonstrated that rapamycin significantly reduced the incidence and severity of the gallbladder carcinoma in a transgenic animal model of GBC (BK5.erbB2 mice) in a dose-dependent manner. ${ }^{17}$ Recently, our research group reported the immunohistochemical expression of phospho-mTOR in 128 advanced gallbladder carcinomas and 99 cases of chronic cholecystitis (Wu et al ${ }^{17}$ analyzed $27 \mathrm{GBC}$ and seven normal tissues). ${ }^{16}$ We showed that phospho-mTOR expression is significantly elevated in human gallbladder carcinoma (64\%) compared with non-neoplastic tissues (24\%). mTOR activation was correlated with a worse prognosis in patients with advanced GBC. ${ }^{16}$

As part of the current study, we analyzed the phosphorylation status of p70S6K in human GBC by immunohistochemistry. p70S6K is probably one of the best characterized downstream effectors of mTORC1. This cytoplasmic serine/threonine kinase is phosphorylated and activated by $\mathrm{mTOR}$ in mitogenic pathways downstream from $\mathrm{PI} 3 \mathrm{~K}$, and is mainly known to regulate protein translation through phosphorylation of the 40S ribosomal protein $\mathrm{S} 6 .{ }^{23}$ Elevated levels or activation of S6K have been associated with several cancers ${ }^{43-51}$ and resistance to chemotherapeutic 
A G-415

LY294002

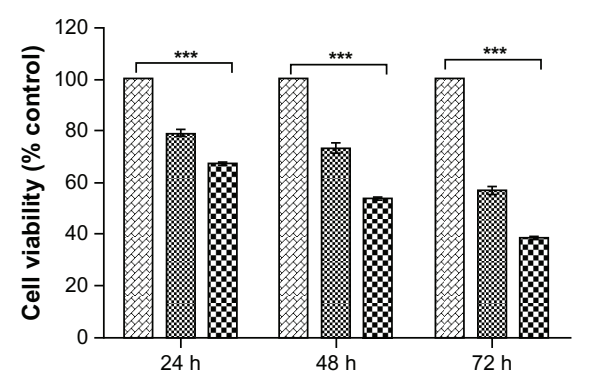

Rapamycin

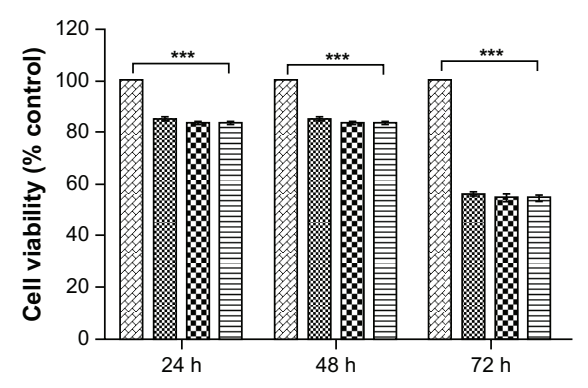

RAD001

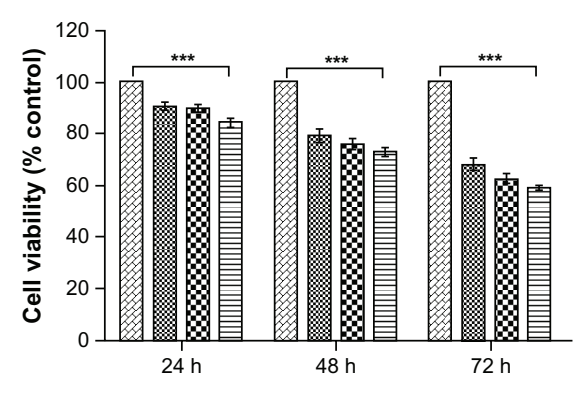

AZD8055

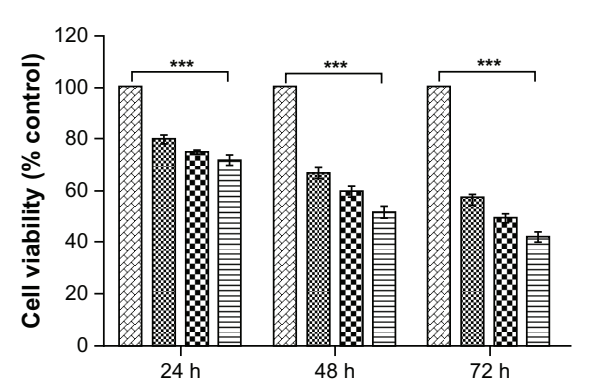

B TGBC-2TKB

LY294002
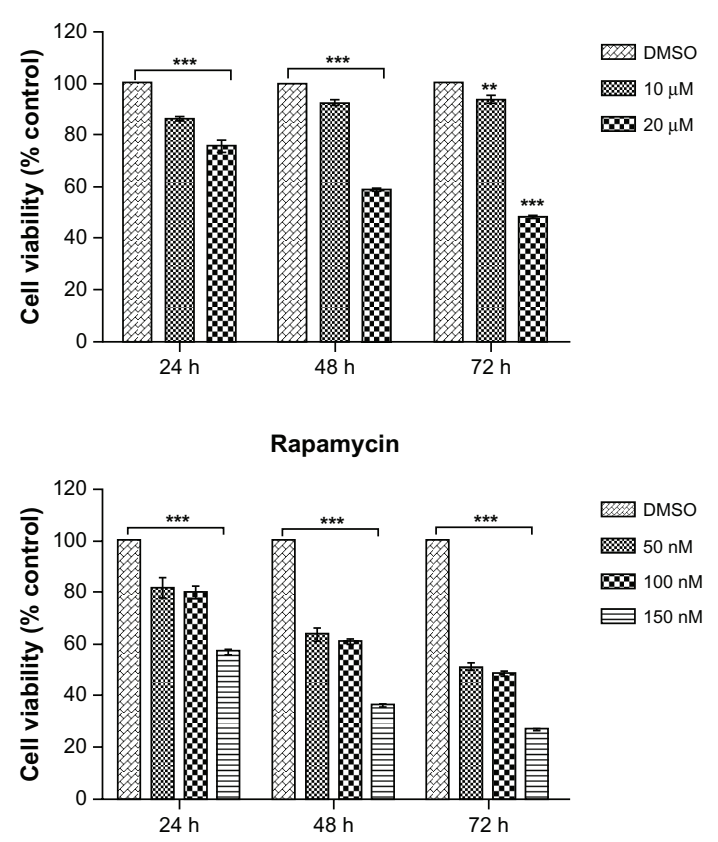

RAD001

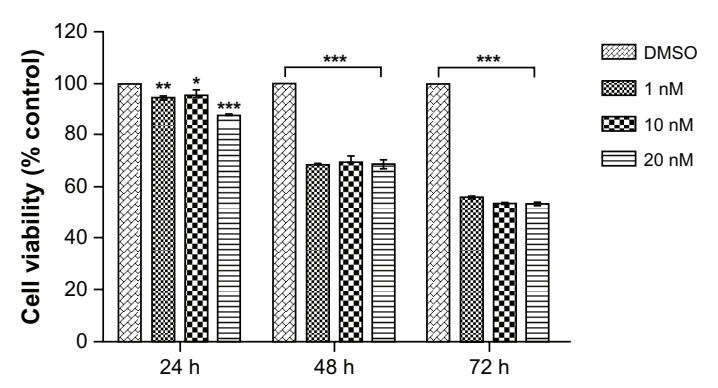

AZD8055

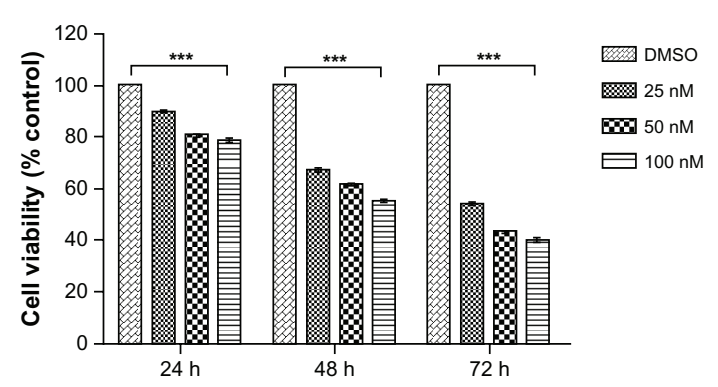

Figure 4 Effect of mTOR inhibitors on cell growth. G-4I5 (A) and TGBC-2TKB (B) cells were treated with LY294002, rapamycin, RAD00I, and AZD8055 at shown concentrations.

Notes: Cell viability was measured after 24,48 , and 72 hours of treatment using CellTiter $96^{\circledR}$ AQueous One Solution Reagent assay. Viable cells are expressed as the percentage of control (untreated cells; $0.01 \%$ dimethylsulfoxide). Values represent the mean \pm standard error of the mean of at least three independent experiments in triplicate. $* P<0.05 ; * * P<0.01$, $* * * P<0.001$ versus control.

Abbreviations: DMSO, dimethyl sulfoxide; mTOR, mammalian target of rapamycin.

drugs. ${ }^{40,52-54}$ As a downstream effector of mTOR pathway, the phosphorylation status of p70S6K is commonly used as a marker of mTOR activity and for pharmacodynamic monitoring of mTOR inhibition. ${ }^{24}$ In this study, we found strong positive staining for phospho-p70S6K in gallbladder tumors
$(88.3 \%, 121 / 137)$. We also observed an elevated expression in dysplasia $(66.7 \%, 12 / 18)$, and there was a significantly difference in the expression of phospho-p70S6K between dysplasia and advanced cancer. The level of phospho-p70S6K did not correlate with any of the evaluated clinicopathological 


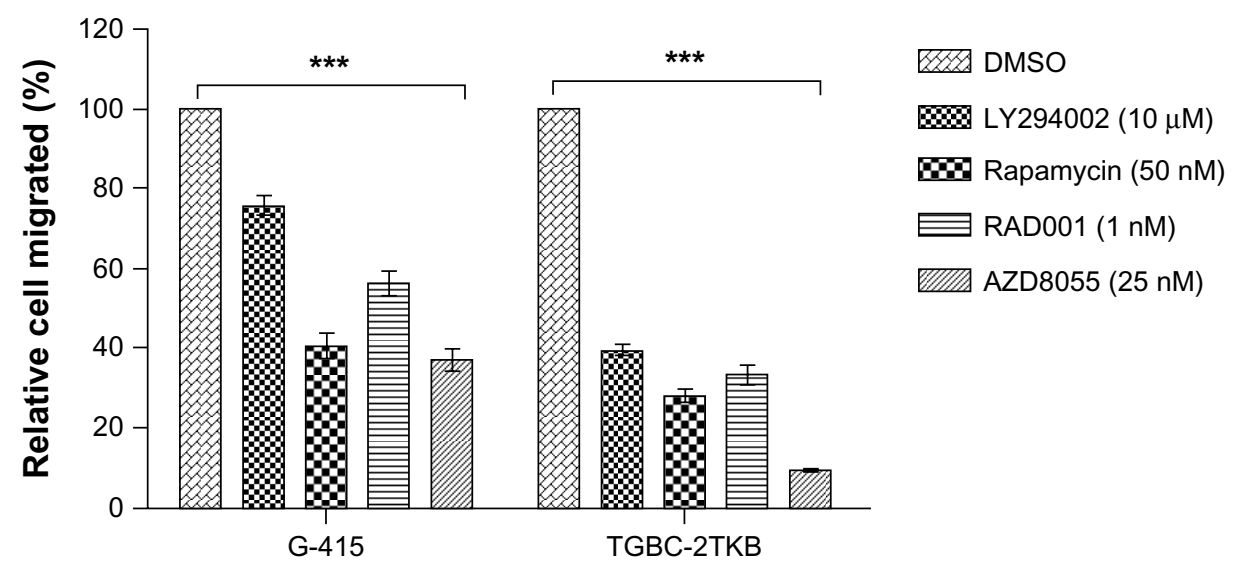

Figure 5 Effect of rapamycin, RAD00I, and AZD8055 on cell migration.

Notes: Assays were performed using 24 -well Transwell ${ }^{\mathrm{TM}}$ plates containing polycarbonate filters with an $8 \mu \mathrm{m}$ pore size. Before seeding, G-4I5 and TGBC-2TKB cells were exposed to LY294002 (I0 $\mu$ M), rapamycin (50 nM), RAD00I (I nM), and AZD8055 (25 nM) for I hour. Dimethylsulfoxide $0.1 \%$ was used as the control. Cells were counted after 24 hours using an optic microscope in 16 randomly selected fields. Results were obtained from three separate experiments and are expressed as the mean \pm standard error of the mean. $* * * P<0.001$ compared with control.

Abbreviation: DMSO, dimethyl sulfoxide.

parameters, which was expected given the high levels of activated p70S6K in most cases.

The phosphorylation status of p70S6K, as well as other components of the mTOR pathway, has already been evaluated in intrahepatic cholangiocarcinoma, another biliary tract carcinoma. Wang et $\mathrm{al}^{49}$ evaluated the immunohistochemical expression of phosphorylated mTOR and its downstream effectors, phospho-p70S6K and phospho-4E-BP1, in 77 intrahepatic cholangiocarcinomas. They found high levels of the three phosphorylated proteins, demonstrating the high prevalence of mTOR pathway activation in these tumors. ${ }^{49}$ Similarly, Lee et al explored the prognostic significance of the AKT1 pathway in a large cohort of intrahepatic cholangiocarcinomas (101 cases). Expression levels of the downstream substrates of mTOR, phospho-p70S6K, and p-4E-BP1 were lower than those reported by Wang et al, but these differences might be due to the $\mathrm{H}$ score cutoff defined by each research group. ${ }^{55}$ Nevertheless, both studies suggest a role of the AKT/ mTOR pathway in intrahepatic cholangiocarcinoma.

On the basis of measuring p70S6K activity, our findings suggest that mTOR signaling is highly active in the early stages of gallbladder carcinogenesis, increasing significantly in advanced GBC. Since both phospho-mTOR and phosphop70S6K are frequently upregulated in patients with advanced GBC, inhibition of the mTOR signaling pathway could be considered a therapeutic strategy in a high proportion of GBC patients.

In addition to the immunohistochemical results, we present here further evidence regarding the activation of this signaling pathway in GBC. Eight GBC cell lines were characterized according to the expression of different components of the AKT/mTOR pathway. Thus, we chose an in vitro model to study the implications of mTOR inhibition using different agents. Based on phospho-AKT expression, G-415, TGBC-1TKB, and TGBC-2TKB could represent an adequate $\mathrm{AKT} / \mathrm{mTOR}$ cell model for in vitro experiments and further in vivo studies using xenograft mouse models. G-415 was originally established from the ascites of a patient with dissemination of GBC, ${ }^{56} \mathrm{TGBC}-2 \mathrm{TKB}$ from a well differentiated tubular adenocarcinoma, and TGBC-1TKB from a poorly differentiated tubular adenocarcinoma in a lymphonode metastasis. ${ }^{57}$ Considering cell culture characteristics (growth rate), mTOR inhibitors were assayed in G-415 and TGBC-2TKB.

We compared the effect of rapamycin, RAD001 (everolimus, a rapamycin analog) and AZD-8055 (an ATP-competitive inhibitor) on the expression of downstream components of the AKT/mTOR pathway and changes in cell growth and migration capability. The results showed that the three agents induced partial cell growth inhibition of both cell lines $(P<$ 0.001 , Figure 4$)$ in a dose-dependent manner. The in vitro migration capability was significantly affected by all drugs $(P<0.001)$, although TGB-2TKB cells were more sensitive than G-415. This could be explained, at least in part, by the apparently constitutive activation of the AKT/mTOR pathway in TGB-2TKB which makes them more responsive to these molecular targeted treatments. With respect to the inhibitors used in this study, the inhibitory effect of AZD8055 on cell migration was more effective than that of rapamycin and RAD001. AZD8055 binds to the mTOR kinase domain and therefore effectively inhibits both mTORC1 and $\mathrm{mTORC} 2$ and has a more profound effect on mTORC1 than rapamycin. ${ }^{35}$

The role of p70S6K activity in cancer includes promotion of both cell growth and migration. Ip et al showed a 
role for $\mathrm{p} 70 \mathrm{~S} 6 \mathrm{~K}$ in the directed migration of human ovarian cancer cells through its ability to directly and indirectly reorganize the actin cytoskeleton via its actin filament cross-linking and Rac1/Cdc42-activating activities. ${ }^{58}$ Given that $\mathrm{p} 70 \mathrm{~S} 6 \mathrm{~K}$ has a well-established role in protein synthesis, this interaction may also have significance in synthesizing local proteins important for propagating the migratory response.

Targeting multiple sites in the PI3K pathway is the current research focus for the development of new anticancer therapies, mainly due to the limited spectrum of clinical response observed with single-agent therapy. This may be due in part to the presence of numerous negative feedback mechanisms on mTOR signaling that can lead to activation of upstream proteins, including AKT. ${ }^{42}$ For this reason, novel agents that target different components of the mTOR pathway, including AKT, p70S6K, 4E-BP1, and phosphoinositidedependent protein kinase 1 , are under investigation in preclinical and clinical studies in other tumors. Considering the high expression of phospho-p70S6K in gallbladder carcinomas, this enzyme could be an interesting target for highly specific cancer therapy. Recently, a new dual inhibitor of AKT/p70S6K has been evaluated in a Phase I clinical trial with patients affected by refractory solid tumors. The drug, LY2780301, is a highly potent, selective, and orally bioavailable ATP-competitive inhibitor against p70S6 kinase and AKT. Preliminary results indicate that LY2780301 has a favorable safety profile and high pharmacokinetic exposures in the range of efficacious doses. ${ }^{59}$

\section{Conclusion}

Despite recent advances in cancer therapy and increased knowledge about cancer biology, the prognosis of patients with GBC remains very poor. To date, several Phase I/II studies have evaluated the efficiency of combined therapy in unresectable, advanced biliary tract carcinomas using classic chemotherapy agents and specific molecularly targeted drugs. However, there is no conclusive evidence that these approaches are more effective than the standard chemotherapy regimen. Therefore, there is an urgent need to develop novel therapeutic alternatives for the treatment of GBC. Our present findings confirm mTOR pathway activation in GBC tumors according to immunohistochemical analysis of phosphorylated p70S6K and in vitro assays in GBC cell lines. The high frequency of upregulated p70S6K $(>80 \%)$ reported in this study suggests that a high proportion of GBC patients might benefit from mTOR pathway-targeted therapies, and also provides a potential novel therapeutic target.

\section{Acknowledgments}

This work was supported by FONDECYT (National Fund for Scientific and Technological Development) (grant 1090171) and DIUFRO (Research Office of Universidad de La Frontera) (project DI11-0039).

\section{Disclosure}

The authors report no conflicts of interest in this work.

\section{References}

1. Lazcano-Ponce EC, Miquel JF, Munoz N, et al. Epidemiology and molecular pathology of gallbladder cancer. CA Cancer J Clin. 2001;51: 349-364.

2. Randi G, Franceschi S, La Vecchia C. Gallbladder cancer worldwide: geographical distribution and risk factors. Int J Cancer. 2006;118: 1591-1602.

3. Kresl JJ, Schild SE, Henning GT, et al. Adjuvant external beam radiation therapy with concurrent chemotherapy in the management of gallbladder carcinoma. Int J Radiat Oncol Biol Phys. 2002;52:167-175.

4. Malik IA, Aziz Z, Zaidi SH, Sethuraman G. Gemcitabine and cisplatin is a highly effective combination chemotherapy in patients with advanced cancer of the gallbladder. Am J Clin Oncol. 2003;26:174-177.

5. Zhu AX, Hong TS, Hezel AF, Kooby DA. Current management of gallbladder carcinoma. Oncologist. 2010;15:168-181.

6. Valle J, Wasan H, Palmer DH, et al. Cisplatin plus gemcitabine versus gemcitabine for biliary tract cancer. N Engl J Med. 2010;362: 1273-1281.

7. Furuse J, Kasuga A, Takasu A, Kitamura H, Nagashima F. Role of chemotherapy in treatments for biliary tract cancer. $J$ Hepatobiliary Pancreat Sci. 2010;19:337-341.

8. Faris JE, Zhu AX. Targeted therapy for biliary tract cancers. J Hepatobiliary Pancreat Sci. 2012;19:326-336.

9. Marino D, Leone F, Cavalloni G, Cagnazzo C, Aglietta M. Biliary tract carcinomas: from chemotherapy to targeted therapy. Crit Rev Oncol Hematol. 2013;85:136-148.

10. Lubner SJ, Mahoney MR, Kolesar JL, et al. Report of a multicenter phase II trial testing a combination of biweekly bevacizumab and daily erlotinib in patients with unresectable biliary cancer: a Phase II Consortium study. J Clin Oncol. 2010;28:3491-3497.

11. Zhu AX, Meyerhardt JA, Blaszkowsky LS, et al. Efficacy and safety of gemcitabine, oxaliplatin, and bevacizumab in advanced biliary-tract cancers and correlation of changes in 18-fluorodeoxyglucose PET with clinical outcome: a phase 2 study. Lancet Oncol. 2010;11:48-54.

12. Bengala C, Bertolini F, Malavasi N, et al. Sorafenib in patients with advanced biliary tract carcinoma: a phase II trial. Br J Cancer. 2010;102: 68-72.

13. Chiorean EG, Ramasubbaiah R, Yu M, et al. Phase II trial of erlotinib and docetaxel in advanced and refractory hepatocellular and biliary cancers: Hoosier Oncology Group GI06-101. Oncologist. 2012;17:13.

14. Lee J, Park SH, Chang HM, et al. Gemcitabine and oxaliplatin with or without erlotinib in advanced biliary-tract cancer: a multicentre, openlabel, randomised, phase 3 study. Lancet Oncol. 2012;13:181-188.

15. Gruenberger B, Schueller J, Heubrandtner U, et al. Cetuximab, gemcitabine, and oxaliplatin in patients with unresectable advanced or metastatic biliary tract cancer: a phase 2 study. Lancet Oncol. 2010;11: 1142-1148.

16. Leal P, Garcia P, Sandoval A, et al. Immunohistochemical expression of phospho-mTOR is associated with poor prognosis in patients with gallbladder adenocarcinoma. Arch Pathol Lab Med. 2013;137:552-557.

17. Wu Q, Kiguchi K, Kawamoto T, et al. Therapeutic effect of rapamycin on gallbladder cancer in a transgenic mouse model. Cancer Res. 2007;67:3794-3800.

18. ClinicalTrials.gov, a serive of the US National Institutes of Health. Available from: http://clinicaltrails.gov/ct2/home. Accessed January, 2013. 
19. National Cancer Institute. MK2206 in Treating Patients With Advanced Refractory Biliary Cancer That Cannot Be Removed by Surgery. In: ClinicalTrials.gov [website on the Internet]. Bethesda, MD: US National Library of Medicine; 2011 [updated May 28, 2013]. Available from: http://clinicaltrials.gov/show/NCT01425879. NLM identifier: NCT01425879. Accessed August 28, 2013.

20. Guertin DA, Sabatini DM. The pharmacology of mTOR inhibition. $S c i$ Signal. 2009;2:pe24.

21. Zaytseva YY, Valentino JD, Gulhati P, Evers BM. mTOR inhibitors in cancer therapy. Cancer Lett. 2012;319:1-7.

22. Carnero A. The PKB/AKT pathway in cancer. Curr Pharm Des. 2010;16:34-44.

23. Hay N, Sonenberg N. Upstream and downstream of mTOR. Genes Dev. 2004;18:1926-1945.

24. Hartmann B. p70S6 kinase phosphorylation for pharmacodynamic monitoring. Clin Chim Acta. 2012;413:1387-1390.

25. Altomare DA, Wang HQ, Skele KL, et al. AKT and mTOR phosphorylation is frequently detected in ovarian cancer and can be targeted to disrupt ovarian tumor cell growth. Oncogene. 2004;23:5853-5857.

26. Gao N, Flynn DC, Zhang Z, et al. G1 cell cycle progression and the expression of G1 cyclins are regulated by PI3K/AKT/mTOR/ p70S6K1 signaling in human ovarian cancer cells. Am J Physiol Cell Physiol. 2004;287:C281-C291.

27. Gao N, Zhang Z, Jiang BH, Shi X. Role of PI3K/AKT/mTOR signaling in the cell cycle progression of human prostate cancer. Biochem Biophys Res Commun. 2003;310:1124-1132.

28. Meng Q, Xia C, Fang J, Rojanasakul Y, Jiang BH. Role of PI3K and AKT specific isoforms in ovarian cancer cell migration, invasion and proliferation through the p70S6K1 pathway. Cell Signal. 2006;18: 2262-2271.

29. Zhou X, Tan M, Stone Hawthorne V, et al. Activation of the Akt/mammalian target of rapamycin/4E-BP1 pathway by ErbB2 overexpression predicts tumor progression in breast cancers. Clin Cancer Res. 2004;10:6779-6788.

30. Keck S, Glencer AC, Rugo HS. Everolimus and its role in hormoneresistant and trastuzumab-resistant metastatic breast cancer. Future Oncol. 2012;8:1383-1396.

31. Mabuchi S, Altomare DA, Cheung M, et al. RAD001 inhibits human ovarian cancer cell proliferation, enhances cisplatin-induced apoptosis, and prolongs survival in an ovarian cancer model. Clin Cancer Res. 2007;13:4261-4270.

32. Tai S, Sun Y, Liu N, et al. Combination of RAD001 (everolimus) and propachlor synergistically induces apoptosis through enhanced autophagy in prostate cancer cells. Mol Cancer Ther. 2012;11 1320-1331.

33. Houghton PJ. Everolimus. Clin Cancer Res. 2010;16:1368-1372.

34. Keniry M, Parsons R. mTOR inhibition, the second generation: ATPcompetitive mTOR inhibitor initiates unexpected receptor tyrosine kinase-driven feedback loop. Cancer Discov. 2011;1:203-204.

35. Chresta CM, Davies BR, Hickson I, et al. AZD8055 is a potent, selective, and orally bioavailable ATP-competitive mammalian target of rapamycin kinase inhibitor with in vitro and in vivo antitumor activity. Cancer Res. 2010;70:288-298.

36. Giubellino A, Bullova P, Nolting S, et al. Combined inhibition of $\mathrm{mTORC} 1$ and $\mathrm{mTORC} 2$ signaling pathways is a promising therapeutic option in inhibiting pheochromocytoma tumor growth: in vitro and in vivo studies in female athymic nude mice. Endocrinology. 2013;154: 646-655.

37. Holt SV, Logie A, Davies BR, et al. Enhanced apoptosis and tumor growth suppression elicited by combination of MEK (selumetinib) and mTOR kinase inhibitors (AZD8055). Cancer Res. 2012;72: 1804-1813.

38. Sini P, James D, Chresta C, Guichard S. Simultaneous inhibition of mTORC 1 and mTORC 2 by mTOR kinase inhibitor AZD 8055 induces autophagy and cell death in cancer cells. Autophagy. 2010;6: $553-554$.
39. Garcia P, Leal P, Alvarez H, et al. Connective tissue growth factor immunohistochemical expression is associated with gallbladder cancer progression. Arch Pathol Lab Med. 2013;137:245-250.

40. Esteva FJ, Guo H, Zhang S, et al. PTEN, PIK3CA, p-AKT, and p-p70S6K status: association with trastuzumab response and survival in patients with HER2-positive metastatic breast cancer. Am J Pathol. 2010;177:1647-1656.

41. Shousha S. Oestrogen receptor status of breast carcinoma: Allred/H score conversion table. Histopathology. 2008;53:346-347.

42. Laplante M, Sabatini DM. mTOR signaling at a glance. J Cell Sci. 2009;122:3589-3594.

43. Dai B, Kong YY, Ye DW, et al. Activation of the mammalian target of rapamycin signalling pathway in prostate cancer and its association with patient clinicopathological characteristics. BJU Int. 2009;104: 1009-1016.

44. Egervari G, Mark A, Hajdu M, et al. Mitotic lymphoma cells are characterized by high expression of phosphorylated ribosomal S6 protein. Histochem Cell Biol. 2011;135:409-417.

45. Heinonen H, Nieminen A, Saarela M, et al. Deciphering downstream gene targets of PI $3 \mathrm{~K} / \mathrm{mTOR} / \mathrm{p} 70 \mathrm{~S} 6 \mathrm{~K}$ pathway in breast cancer. $B M C$ Genomics. 2008;9:348.

46. Lang SA, Gaumann A, Koehl GE, et al. Mammalian target of rapamycin is activated in human gastric cancer and serves as a target for therapy in an experimental model. Int J Cancer. 2007;120:1803-1810.

47. Miyakawa M, Tsushima T, Murakami H, et al. Increased expression of phosphorylated p70S6 kinase and Akt in papillary thyroid cancer tissues. Endocr J. 2003;50:77-83.

48. No JH, Jeon YT, Park IA, et al. Activation of mTOR signaling pathway associated with adverse prognostic factors of epithelial ovarian cancer. Gynecol Oncol. 2011;121:8-12.

49. Wang Z, Zheng T, Wu Q, Wang J, Wu C. Immunohistochemical analysis of the mTOR pathway in intrahepatic cholangiocarcinoma. Neoplasma. 2012;59:137-141

50. Xiao L, Wang YC, Li WS, Du Y. The role of mTOR and phosphop70S6K in pathogenesis and progression of gastric carcinomas: an immunohistochemical study on tissue microarray. J Exp Clin Cancer Res. 2009;28:152.

51. Zhou HY, Wong AS. Activation of p70S6K induces expression of matrix metalloproteinase 9 associated with hepatocyte growth factormediated invasion in human ovarian cancer cells. Endocrinology. 2006; 147:2557-2566.

52. Dhar R, Basu A. Constitutive activation of p70S6 kinase is associated with intrinsic resistance to cisplatin. Int J Oncol. 2008;32: $1133-1137$.

53. Kim EK, Kim HA, Koh JS, et al. Phosphorylated S6K1 is a possible marker for endocrine therapy resistance in hormone receptor-positive breast cancer. Breast Cancer Res Treat. 2011;126:93-99.

54. Liu LZ, Zhou XD, Qian G, et al. AKT1 amplification regulates cisplatin resistance in human lung cancer cells through the mammalian target of rapamycin/p70S6K1 pathway. Cancer Res. 2007;67:6325-6332.

55. Lee D, Do IG, Choi K, et al. The expression of phospho-AKT1 and phospho-MTOR is associated with a favorable prognosis independent of PTEN expression in intrahepatic cholangiocarcinomas. Mod Pathol. 2012;25:131-139.

56. Koyama S, Yoshioka T, Mizushima A, et al. Establishment of a cell line (G-415) from a human gallbladder carcinoma. Gann. 1980;71:574-575.

57. Ghosh M, Koike N, Yanagimoto G, et al. Establishment and characterization of unique human gallbladder cancer cell lines. Int J Oncol. 2004;24:1189-1196.

58. Ip CK, Cheung AN, Ngan HY, Wong AS. p70S6 kinase in the control of actin cytoskeleton dynamics and directed migration of ovarian cancer cells. Oncogene. 2011;30:2420-2432.

59. Calvo et al. First-in-human phase I study of LY2780301, an oral P70S6K/AKT inhibitor, in patients with refractory solid tumors. J Clin Oncol. 2012;30 Suppl:Abstr 3005. 


\section{Supplementary materials}

Table SI Association of phospho-p70S6K expression status (positive/negative) and clinicopathological parameters

\begin{tabular}{|c|c|c|c|c|c|c|c|c|c|c|}
\hline \multirow[t]{2}{*}{ Category } & \multirow[t]{2}{*}{ Subcategory } & \multicolumn{3}{|c|}{ Dysplasia $(n=18)$} & \multicolumn{3}{|c|}{ Early cancer $(n=26)$} & \multicolumn{3}{|c|}{ Advanced cancer $(n=137)$} \\
\hline & & Positive & Negative & $P$-value & Positive & Negative & $P$-value & Positive & Negative & $P$-value \\
\hline \multirow[t]{2}{*}{ Age } & $<65$ & 9 & 4 & 0.71 & 14 & I & $0.28^{*}$ & 62 & 7 & $0.6 I^{*}$ \\
\hline & $\geq 65$ & 3 & 2 & & 8 & 3 & & 59 & 9 & \\
\hline \multirow[t]{2}{*}{ Sex } & Female & 10 & 5 & $>0.99 *$ & 20 & 4 & $>0.99 *$ & 98 & 16 & $0.07 *$ \\
\hline & Male & 2 & I & & 2 & 0 & & 23 & 0 & \\
\hline \multirow[t]{2}{*}{ Ethnicity } & Mapuche & 0 & 0 & & 3 & 0 & $>0.99 *$ & 29 & 3 & $0.76 *$ \\
\hline & Hispanic & 12 & 6 & & 19 & 4 & & 92 & 16 & \\
\hline Histological & Moderate & & & & 8 & 0 & 0.06 & 13 & 2 & 0.53 \\
\hline \multirow[t]{2}{*}{ differentiation** } & Well & & & & 6 & 1 & & 66 & 6 & \\
\hline & Poor & & & & 7 & I & & 36 & 6 & \\
\hline
\end{tabular}

Notes: P-value for the chi-square test; *Fisher's exact test. **Three (early cancer) and eight (advanced cancer) cases with missing information were excluded from analysis.
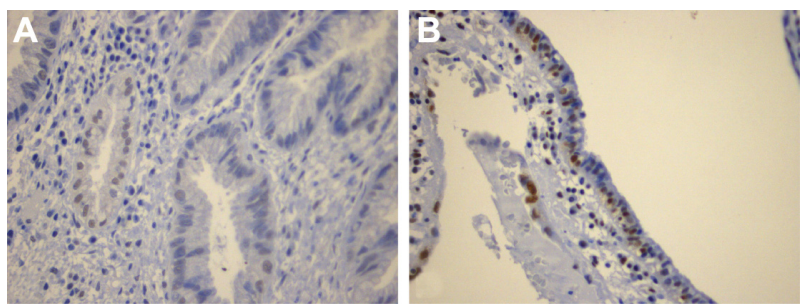

Figure SI Immunohistochemical staining of phospho-p70S6K in chronic cholecystitis. (A) Chronic cholecystitis negative for phospho-p70S6K immunohistochemistry. (B) Chronic cholecystitis showing positive staining of phospho-p70S6K.
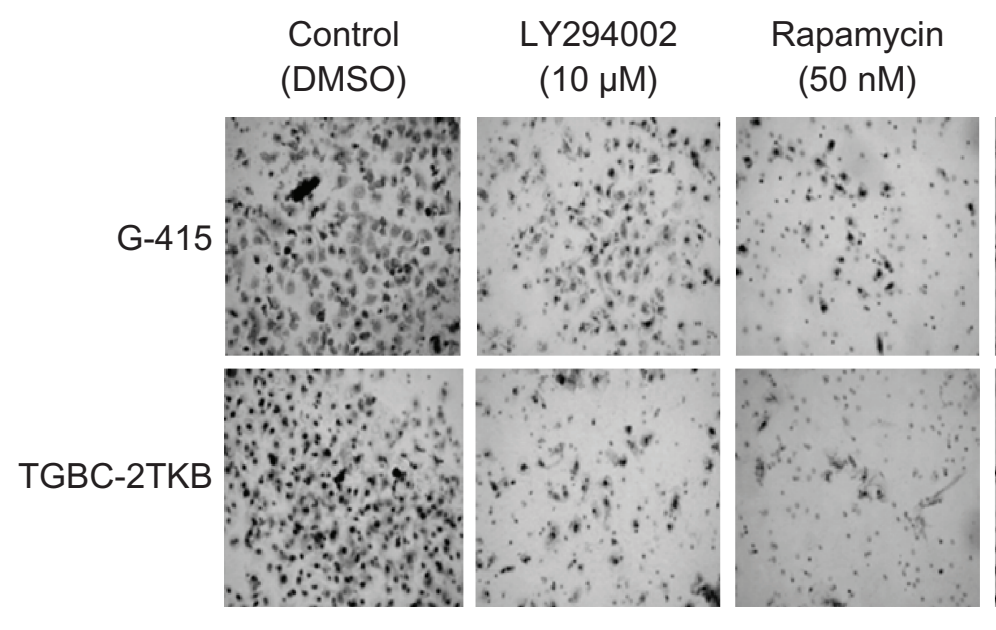

\section{RAD001}

$(1 \mathrm{nM})$
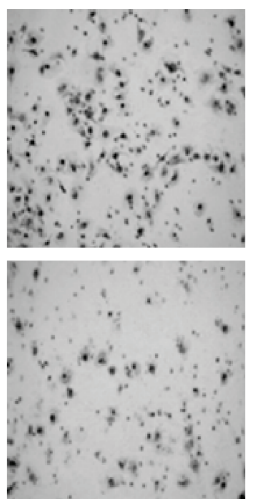

AZD8055

$(25 \mathrm{nM})$
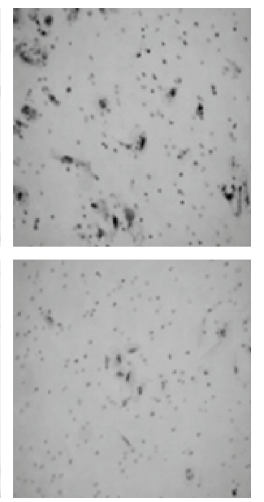

Figure S2 Representative images of the effect of mTOR inhibitors on cell migration. Assays were performed using 24-well Transwell ${ }^{\mathrm{TM}}$ plates containing polycarbonate filters with an $8 \mu \mathrm{m}$ pore size. Before seeding, G-4I5 and TGBC-2TKB cells were exposed to LY294002 (I0 $\mu$ M), rapamycin (50 nM), RAD00I (I nM), and AZD8055 (25 nM) for I hour. Dimethylsulfoxide (DMSO) $0.1 \%$ was used as control. Cells were counted after 24 hours using an optic microscope.

Abbreviations: DMSO, dimethyl sulfoxide; mTOR, mammalian target of rapamycin.

OncoTargets and Therapy

\section{Publish your work in this journal}

OncoTargets and Therapy is an international, peer-reviewed, open access journal focusing on the pathological basis of all cancers, potential targets for therapy and treatment protocols employed to improve the management of cancer patients. The journal also focuses on the impact of management programs and new therapeutic agents and protocols on

\section{Dovepress}

patient perspectives such as quality of life, adherence and satisfaction. The manuscript management system is completely online and includes a very quick and fair peer-review system, which is all easy to use. Visit http://www.dovepress.com/testimonials.php to read real quotes from published authors. 